\title{
ARTICLE \\ Measuring novel antecedents of mental illness: the Questionnaire of Unpredictability in Childhood
}

Laura M. Glynn ${ }^{1,2}$, Hal S. Stern ${ }^{3}$, Mariann A. Howland ${ }^{4}$, Victoria B. Risbrough $\mathbb{i D}^{5,6}$, Dewleen G. Baker ${\left(\mathbb{I D}^{5,6}\right.}^{5}$ Caroline M. Nievergelt ${ }^{5,6}$, Tallie Z. Baram ${ }^{7,8,9}$ and Elysia P. Davis ${ }^{2,10}$

Increasing evidence indicates that, in addition to poverty, maternal depression, and other well-established factors, unpredictability of maternal and environmental signals early in life influences trajectories of brain development, determining risk for subsequent mental illness. However, whereas most risk factors for later vulnerability to mental illness are readily measured using existing, clinically available tools, there are no similar measures for assessing early-life unpredictability. Here we validate the Questionnaire of Unpredictability in Childhood (QUIC) and examine its associations with mental health in the context of other indicators of childhood adversity (e.g., traumatic life events, socioeconomic status, and parenting quality). The QUIC was initially validated through administration to a cohort of adult females $(N=116)$ and then further refined in two additional independent cohorts (male Veterans, $N=95$, and male and female adolescents, $N=175)$. The QUIC demonstrated excellent internal $(a=0.89)$ and test-retest reliability $(r=92)$. Scores on the QUIC were positively correlated with other prospective indicators of exposures to unpredictable maternal inputs in infancy and childhood (unpredictable maternal mood and sensory signals), and accuracy of recall also was confirmed with prospective data. Importantly, the QUIC predicted symptoms of anxiety, depression, and anhedonia in the three study cohorts, and these effects persisted after adjusting for other previously established risk factors. The QUIC, a reliable and valid self-report assessment of exposure to unpredictability in the social, emotional, and physical domains during early life, is a brief, comprehensive, and promising instrument for predicting risk for mental illness.

Neuropsychopharmacology (2019) 44:876-882; https://doi.org/10.1038/s41386-018-0280-9

\section{INTRODUCTION}

It is widely recognized that the family and household are the primary social, emotional, and physical contexts in which child development unfolds [1, 2]. It is therefore not surprising that earlylife experiences such as living in poverty, trauma exposure, and harsh parental care exert persisting influences on lifespan trajectories of mental health [3-5]. Although known childhood influences such as these do explain a significant proportion of the variation in mental health risk, there remains a significant gap in our ability to predict who will develop vulnerability or resistance to mental illness.

Recently, a novel conceptualization of early-life adversityunpredictability in the environment-has emerged that may shed additional light on risk for later mental illness and its underlying mechanisms. The importance of unpredictability of sensory inputs is supported by animal models demonstrating that fragmented or unpredictable maternal signals influence the maturation of systems governing emotional and cognitive function in the developing brain $[6,7]$. For example, when rodents are exposed to unpredictable maternal signals early in life, these rats exhibit diminished memory function $[8,9]$ and reduced ability to experience pleasure (anhedonia) beginning in adolescence
[10-12]. Further, the predictability of these maternal signals influences synaptic growth and persistence in cognitive and emotional brain circuits, which then affect the maturation of connectivity in these circuits $[13,14]$. Importantly, these effects are independent of the quantity and quality of the maternal signals, underscoring the importance of unpredictability of these inputs $[10,15]$

Complementary conceptual models also recognize that, when characterizing early-life adversity, in addition to harshness or cumulative stress exposures, unpredictability represents a primary and unique determinant of development $[16,17]$. Consistent with this view, there is evidence in humans that exposure to unpredictability in social, emotional, and physical domains in early life influences developmental trajectories. For example, mother-child dyadic synchrony is a positive predictor of more optimal child development $[18,19]$, and it is well established that a hallmark of secure attachment is the receipt of reliable and appropriate maternal care [20]. Similarly, more residential transitions, changes in parental occupation status, less routinization in the family unit, and more chaos in the household each have been linked to poorer child mental health [21-24]. In addition, new findings specifically indicate that unpredictable patterns of both

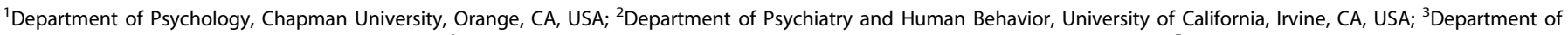

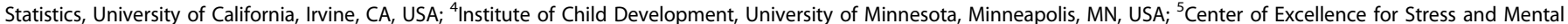

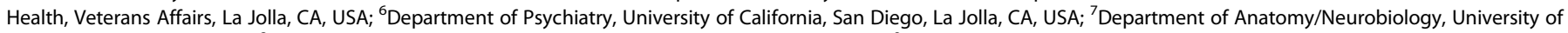

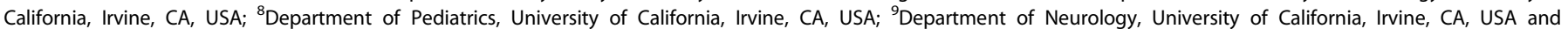
${ }^{10}$ Department of Psychology, University of Denver, Denver, CO, USA

Correspondence: Laura M. Glynn (Iglynn@chapman.edu)

Received: 13 June 2018 Revised: 6 November 2018 Accepted: 10 November 2018

Published online: 23 November 2018 
maternal behavior and mood, independent of its quality or valence (e.g., maternal sensitivity or level of depressive symptoms), uniquely predicts child mental health risk [15, 25].

Despite the emerging evidence from both animal models and humans indicating that unpredictability of inputs shapes the developing brain and affects risk trajectories of mental health, no instrument exists to provide a comprehensive assessment of exposures to unpredictability in childhood. The primary goal of this study was to evaluate the Questionnaire of Unpredictability in Childhood (QUIC) - a self-report measure that assesses predictability (social, emotional, and physical) in the childhood environment. To this end, we examined the psychometric properties (internal consistency and test-retest reliability) of the QUIC in three independent cohorts. We also determined its construct validity by examining its associations with prospectively assessed aspects of the childhood environment. The second goal of the study was to test the hypothesis that increased exposures to unpredictability in childhood, determined by the QUIC, would predict increased mental health symptoms.

\section{METHODS}

Overview

An initial set of items were generated by a subset of the authors (EPD, LMG) for inclusion in the QUIC. The items were intended to reflect unpredictability in various aspects of a child's environment (social, emotional, and physical domains). Data were collected in three independent cohorts to refine the scale and determine its reliability and validity. Cohort 1 consisted of adult females $(N=$ 116), Cohort 2 of male Veterans $(N=95)$, and Cohort 3 of male and female adolescents $(N=175)$. In addition to completion of the QUIC, participants completed other instruments to assess reliability and validity.

\section{Description of scale}

The QUIC is a series of items that respondents endorse as applying to their life prior to age 18 , with a subset of questions focusing specifically on events more likely to occur in earlier childhood (prior to age 12). Items were developed by the authors and then further refined with conceptual and empirical analyses. Examples include "I experienced changes in my custody arrangement", "At least one of my parents regularly checked that I did my homework", and "At least one of my parents was disorganized." The initial version comprised 43 items which are endorsed as either "yes" or "no" (some of which are reverse coded). All items were evaluated for clarity, endorsement rates, correlation with related items, and fit to subscales, with five items ultimately eliminated. The final scale consists of 38 items and 5 subscales (Table 1): Parental Involvement (9 items), Parental Predictability (12 items), Parental Environment (7 items), Physical Environment (7 items), and Safety and Security ( 3 items). Scores on the scale can range from 0 to 38 , with a higher score indicating greater exposure to unpredictability in the environment prior to 18 years of age. More detailed information about scale development is given in Supplemental Materials.

\section{Description of study cohorts}

Cohort 1: Adult females. The cohort of adult females consisted of 116 women participating in an ongoing, longitudinal study of maternal and child health (see Table 2 for demographics). In addition to completing the QUIC, women completed the Trauma History Questionnaire (THQ) [26], which consists of 24 yes/no items that address lifetime traumatic events related to crime, general disaster, and trauma, and physical and sexual experiences. For the purposes of comparison with the QUIC, items endorsed as occurring prior to the age of 18 were summed to create a childhood traumatic life events score. Mental health risk in this cohort was assessed with the 10-item Edinburgh Postnatal
Depression Scale [27] at 6 months postpartum. Current family income-to-needs ratio also was considered and was calculated by dividing the total annual household income by the appropriate $U$. S. Census Bureau poverty threshold based on family size. To evaluate temporal stability of the QUIC, a subset of these women $(n=58)$ completed the measure twice (mean time between assessments $=13.6$ weeks). The protocol was approved by the institutional review board (IRB) and all participants provided written and informed consent.

Cohort 2: Male Veterans. The QUIC was administered to 95 male military Veterans (See Table 2 for demographics). Veterans were either participating in a follow-up assessment from a prospective, longitudinal study of deployment trauma in male Marines and Navy Corpsmen [28] or were contributing data to the Center of Excellence for Stress and Mental Health TBI/PTSD biorepository data archives, which collects biological samples and traumarelated mental health information in treatment-seeking Veterans within the San Diego Veterans Affairs Healthcare System. Both protocols were approved by the Veterans Affairs IRBs, and all participants provided written and informed consent. In addition to the QUIC, the Veterans also completed the 28-item Childhood Trauma Questionnaire (CTQ) [29], which assesses the severity of childhood emotional abuse and neglect, physical abuse and neglect, and sexual abuse. Mental health risk was assessed with the 22-item Anhedonic Depression scale of the Mood and Anxiety Symptom Questionnaire (MASQ-AD) [30] This scale measures symptoms of low positive emotion and anhedonia considered to be specific to depression and has been utilized independently of the full MASQ scale [31].

Cohort 3: Adolescents. This study cohort consisted of 175 adolescents (52\% female) and their mothers participating in an ongoing, longitudinal study of maternal and child health (see Table 2 for demographics). These mother-child pairs have been followed prospectively from the prenatal period through adolescence. The study protocol was approved by the institutional IRB and written and informed consent was obtained from the mothers and assent from the children beginning at age 7 .

Assessments during infancy and childhood: Unpredictability of maternal sensory signals was measured when the children were 6 and 12 months of age. Briefly, maternal auditory, tactile, and visual signals were coded from videos of 10-min mother-child play periods. Predictability of sensory signals was characterized for each video using entropy rate as detailed in Davis et al. [15]. The predictability measure used here is the average of the entropy rate at 6 and 12 months, with missing values imputed via regression. Predictability of maternal mood was measured in infancy (at 3, 6, 12 , and 24 months) and childhood (at 5, 6, and 9 years) as described in Glynn et al. [25]. Scores during infancy and childhood were separately averaged.

Assessments in adolescence: Adolescents (mean age $=13.7$ ) completed the QUIC and measures of mental health risk. Adolescents reported on their anxiety symptoms using the 20item trait anxiety scale from the State-Trait Anxiety Inventory for Children [32] and depressive symptoms with the 12-item Children's Depression Inventory [33]. As in the cohort of male Veterans, symptoms of anhedonia were measured with the MASQ$\mathrm{AD}$. Adolescents additionally reported on parenting style using the Alabama Parenting Questionnaire (APQ) [34], which assesses five domains of parenting quality including consistency of discipline and parental supervision. At the concurrent assessment point, mothers provided demographic information and reported on their adolescents' life events using the Coddington Life Events Scale for children (CLES) [35]. They also completed the 15-item Chaos, Hubbub and Order Scale (CHAOS) [36], which assesses 
Table 1. QUIC items by subscale

Parental monitoring and involvement

Prior to age 12: I had a set morning routine on school days (i.e., I usually did the same thing each day to get ready). (R)

Prior to age 12: My parents kept track of what I ate (e.g., made sure that I didn't skip meals or tried to make sure I ate healthy food). (R)

Prior to age 12: My family ate a meal together most days. (R)

Prior to age 12: My parents tried to make sure I got a good night's sleep (e.g., I had a regular bedtime, my parents checked to make sure I went to sleep). (R)

Prior to age 12: I had a bedtime routine (e.g., my parents tucked me in, my parents read me a book, I took a bath). (R)

Prior to age 12: In my afterschool or free time hours at least one of my parents knew what I was doing. (R)

Prior to age 12: At least one of my parents regularly checked that I did my homework. (R)

Prior to age 18: At least one of my parents regularly kept track of my school progress. (R)

Prior to age 18: At least one parent made time each day to see how I was doing. (R)

Parental predictability

Prior to age 12: My parents were often late to pick me up (e.g. from school, aftercare or sports)

Prior to age 12: I usually knew when my parents were going to be home. (R)

Prior to age 18: At least one of my parents had punishments that were unpredictable

Prior to age 18: I often wondered whether or not one of my parents would come home at the end of the day

Prior to age 18: My family planned activities to do together. (R)

Prior to age 18: At least one of my parents would plan something for the family, but then not follow through with the plan

Prior to age 18: My family had holiday traditions that we did every year (e.g., cooking a special food at a particular time of year/decorate the house the same way). (R)

Prior to age 18: At least one of my parents was disorganized

Prior to age 18: At least one of my parents was unpredictable

Prior to age 18: For at least one of my parents, when they were upset I did not know how they would act

Prior to age 18: One of my parents could go from calm to furious in an instant

Prior to age 18: One of my parents could go from calm to stressed or nervous in an instant

Parental environment

Prior to age 18: There was a long period of time when I didn't see one of my parents (e.g. military deployment, jail time, custody arrangements) Prior to age 18: I experienced changes in my custody arrangement

Prior to age 18: At least one of my parents changed jobs frequently

Prior to age 18: There were times when one of my parents was unemployed and couldn't find a job even though he/she wanted one

Prior to age 18: My parents had a stable relationship with each other. (R)

Prior to age 18: My parents got divorced

Prior to age 18: At least one of my parents had many romantic partners

Physical environment

Prior to age 18: There were often people coming and going in my house that I did not expect to be there

Prior to age 18: I moved frequently

Prior to age 18: I changed schools frequently

Prior to age 18: I changed schools mid-year

Prior to age 18: I lived in a clean house. (R)

Prior to age 18: I lived in a cluttered house (e.g., piles of stuff everywhere)

Prior to age 18: In my house things I needed were often misplaced so that I could not find them

Safety and security

Prior to age 18: There was a period of time when I often worried that I was not going to have enough food to eat

Prior to age 18: There was a period of time when I often worried that my family would not have enough money to pay for necessities like clothing or bills

Prior to age 18: There was a period of time when I did not feel safe in my home

(R) indicates item is reverse scored

unpredictability and confusion in the home. Family income-toneeds ratio was calculated using the same method described for Cohort 1. Missing data for APQ scales (5.7\%), CLES (5.7\%), CHAOS $(11.4 \%)$, and income-to-needs ratio (1.7\%) were addressed through multiple imputation [37]. Ten complete data sets were generated using the multiple imputation procedure in SPSS (version 23), which uses a series of sequential imputation models [37]. Multiple regression models were fit to each of the complete data sets and the results combined using the standard approach for multiple imputation [38].

\section{RESULTS}

Mean scores and endorsement rates

Means and standard deviations for the overall scale score and five subscales for each of the three cohorts are provided in 
Table 2. Description of study cohorts

\begin{tabular}{llll}
\hline & $\begin{array}{l}\text { Adult females } \\
(N=116)\end{array}$ & $\begin{array}{l}\text { Adult } \\
\text { males } \\
(N=95)\end{array}$ & $\begin{array}{l}\text { Adolescents } \\
(N=175)\end{array}$ \\
\hline $\begin{array}{l}\text { Age (mean years) } \\
\text { Sex (\% female) }\end{array}$ & 30 & 35 & 14 \\
$\begin{array}{l}\text { Race/ethnicity (\%) } \\
\quad \text { Caucasian, non- }\end{array}$ & 35 & 0 & 52 \\
$\quad$ Hispanic & & 63 & 39 \\
$\quad$ Hispanic/Latino(a) & 42 & 14 & 30 \\
$\quad \begin{array}{l}\text { Asian } \\
\text { Black }\end{array}$ & 12 & 5 & 6 \\
$\quad 3$ & 11 & 5 \\
$\begin{array}{l}\text { Multi-ethnic } \\
\text { Highest level of } \\
\text { education (mean } \\
\text { years) }\end{array}$ & 7 & 7 & 21 \\
$\begin{array}{l}\text { Annual household } \\
\text { income (mean USD) }\end{array}$ & 75,634 & 14 & - \\
\hline \begin{tabular}{l} 
Income data not available for adult males \\
\hline
\end{tabular} & & \\
\hline
\end{tabular}

Table 3 (a summary of the distribution of endorsement rates as well as endorsement rates for each item can be found in Table S1). Compared to adult females and adolescents, the male Veterans had higher scores on the overall scale and on 4 of the 5 subscales (all $p s<.01$ ). No differences were observed between male and female adolescents on any of the scale scores. Although scale sum scores differed across cohorts, inspection of the distribution of responses across cohorts (i.e., the probability that a single item was endorsed more or less frequently compared to others within a cohort) did not reveal notable or consistent differences (Table S1).

Evidence for internal reliability

Cronbach's alphas demonstrated strong internal consistency for the overall score across the three cohorts $(a=0.84-0.92$; see Table 4). Internal consistency also was good for the subscales, with the adolescents showing slightly lower alphas than the two adult cohorts. Further, alpha values tend to be smaller for scales with fewer items [39] and that is true here for the three-item Safety and Security subscale.

Evidence for test-retest reliability

Test-retest reliability was excellent, with $r=.92$ for the overall scale score. Temporal stability also was strong for each of the subscales, ranging from .75 to .88 (Table 4 ).

Evidence for validity of retrospective recall

Maternal report data collected prospectively and longitudinally beginning in infancy in the adolescent cohort provides strong evidence for accuracy of reporting on the QUIC. Consistent with prospectively collected data, $83 \%$ of children who endorsed item 20 , "I moved frequently," in fact moved three or more times during their lifetimes, and $99 \%$ of children who reported parental divorce (item 29) had parents who were either legally divorced (93\%) or permanently separated (6\%).

Evidence for construct validity

Evidence for construct validity was provided by associations between the QUIC and the two prospectively assessed, observational measures of exposure to unpredictable maternal inputs. Specifically, adolescents who reported higher scores on the QUIC had been exposed to more unpredictable maternal sensory signals measured in infancy $(r=.23, p<.05)$ and also to more unpredictable maternal mood assessed in both infancy $(r=.17$, $p<.05)$ and childhood $(r=.28, p<.001)$. In addition, higher scores on the QUIC were associated with self-report measures evaluating unpredictability within a specific domain. As shown in Table S2, adolescent reports of more inconsistent discipline and less parental supervision and maternal reports of greater household chaos all were positively associated with QUIC scores. As shown in Table 5, as expected, the QUIC also was positively associated with exposures to traumatic and stressful life events in all three cohorts.

Evidence for predictive validity: QUIC predicts mental health risk The links between the QUIC and mental health indicators are shown in Table 5. Among the adult women, higher scores on the QUIC were associated with elevated postpartum depressive symptoms $(r=.27, p<.05)$, and male Veterans who reported more childhood unpredictability on the QUIC reported higher levels of anhedonic symptoms $(r=.28, p<.01)$. Similarly, adolescents who reported greater childhood unpredictability on the QUIC had elevated symptoms of anhedonia $(r=.37, p<.001)$, depression $(r=.42, p<.001)$, and anxiety $(r=.43, p<.001)$.

To determine whether the QUIC adds predictive power beyond other measures of specific aspects of unpredictability (e.g., household chaos, inconsistent parental discipline) and other previously established indicators of childhood adversity (incometo-needs ratio, life events), we tested the links between adolescent mental health and the QUIC after statistical consideration of these factors. The relation between the QUIC and adolescent anhedonia, depression, and anxiety symptoms remained after adjustment for the APQ scales, INR, household chaos, and child life events supporting its unique predictive validity (see Table 6). In addition, modeling the QUIC with measures of childhood maltreatment and traumatic life events did not substantively alter the relation between the QUIC and anhedonia in the male Veterans $(\beta=0.28$, $p<.05$ vs $\beta=0.34, p<.05$ ) or with depression in the adult females $(\beta=0.30, p<.05$ vs $\beta=0.28, p=.06$; see Table S3 for these regression models).

\section{DISCUSSION}

Accumulating evidence suggests that predictability or patterns of inputs in the social, emotional, and physical environments during development shapes risk for psychopathology, indicating a need for efficient and accurate methods for assessing exposure to unpredictability in early life. To address this gap, we developed the QUIC, which represents the first comprehensive measure of exposures to unpredictability in the social, emotional, and physical environment in childhood. This 38-item scale is easy to administer and contains five subscales. The QUIC exhibits good internal and test-retest reliability and is correlated with prospective and observational indicators of unpredictable maternal sensory signals and maternal mood in childhood, as well as reports of unpredictability in parenting practices and household chaos, providing strong support for its construct validity. It also was positively correlated with other validated and widely used measures of adversity in childhood such as the CTQ [29] and THQ [26].

In addition to demonstrating its sound psychometric properties, we also present data in three independent cohorts indicating that the QUIC not only reliably predicts mental health but also predicts mental health beyond other widely used measures of early adversity. In a cohort of adult females, higher QUIC scores were associated with increased postpartum depressive symptoms. In a group of male Veterans, elevated scores on the QUIC predicted higher levels of anhedonia symptoms. Last, among male and female adolescents, QUIC scores were positively associated with risk for symptoms of anhedonia, anxiety, and depression. Importantly, these relations were not accounted for by other well-established childhood indicators of risk, including income-to- 
Table 3. Means and standard deviations of QUIC score and subscale scores

\begin{tabular}{llllll}
\hline & Adult females $(N=116)$ & Adult males $(N=95)$ & \multicolumn{2}{l}{ Adolescents } \\
\cline { 3 - 5 } & & & All $(N=175)$ & Females $(n=91)$ & Males $(n=84)$ \\
\hline Total QUIC Score (38 items) & $7.7(6.8)$ & $10.8(8.4)$ & $7.4(5.5)$ & $7.3(5.6)$ \\
Parental monitoring and involvement (9 items) & $1.6(2.0)$ & $2.6(2.8)$ & $1.1(1.4)$ & $1.1(1.4)$ & $1.2(1.3)$ \\
Parental predictability (12 items) & $3.2(2.9)$ & $3.9(3.2)$ & $3.4(2.5)$ & $3.4(2.7)$ & $3.4(2.2)$ \\
Parental environment (7 items) & $1.4(1.7)$ & $2.3(2.1)$ & $1.3(1.6)$ & $1.3(1.6)$ & $1.4(1.7)$ \\
Physical environment (7 items) & $1.0(1.4)$ & $1.2(1.6)$ & $1.1(1.4)$ & $1.1(1.4)$ & $1.2(1.5)$ \\
Safety and security (3 items) & $0.4(0.8)$ & $0.9(1.0)$ & $0.4(0.7)$ & $0.5(0.7)$ & $0.3(0.6)$ \\
\hline
\end{tabular}

Table 4. Psychometric properties of the QUIC

\begin{tabular}{lllll}
\hline & Test-retest reliability $(r s)$ & \multicolumn{2}{l}{ Internal consistency (Cronbach's alphas) } \\
\cline { 5 - 6 } & Adult females $(N=58)$ & Adult females $(N=116)$ & Adult males $(N=95)$ & Adolescents $(N=175)$ \\
\hline Total QUIC Score (38 items) & .92 & .90 & .92 & .84 \\
Parental monitoring and involvement (9 items) & .75 & .77 & .87 & .53 \\
Parental predictability (12 items) & .87 & .80 & .83 & .69 \\
Parental environment (7 items) & .88 & .70 & .76 & .70 \\
Physical environment (7 items) & .80 & .68 & .68 & .65 \\
Safety and security (3 items) & .80 & .58 & .56 & .42 \\
\hline
\end{tabular}

Table 5. Associations between the QUIC, early-life adversity, and mental health risk (bivariate correlations)

\begin{tabular}{|c|c|c|c|c|c|c|}
\hline & 1. & 2. & 3. & 4. & 5. & 6. \\
\hline \multicolumn{7}{|l|}{ Adult females } \\
\hline 1. QUIC & - & & & & & \\
\hline $\begin{array}{l}\text { 2. Childhood traumatic } \\
\text { events (THQ) }\end{array}$ & $.26^{+}$ & - & & & & \\
\hline 3. Income-to-needs ratio & -.09 & .08 & - & & & \\
\hline $\begin{array}{l}\text { 4. Depressive symptoms } \\
\text { (EPDS) }\end{array}$ & $.30^{*}$ & .05 & -.19 & - & & \\
\hline \multicolumn{7}{|l|}{ Adult males } \\
\hline 1. QUIC & - & & & & & \\
\hline $\begin{array}{l}\text { 2. Childhood maltreatment } \\
\text { (CTQ) }\end{array}$ & $.61^{* *}$ & - & & & & \\
\hline $\begin{array}{l}\text { 5. Anhedonia symptoms } \\
\text { (MASQ-AD) }\end{array}$ & $.28^{* *}$ & .06 & & & - & \\
\hline \multicolumn{7}{|l|}{ Adolescents } \\
\hline 1. QUIC & - & & & & & \\
\hline $\begin{array}{l}\text { 2. Childhood life events } \\
\text { (CLES) }\end{array}$ & $.37^{* *}$ & - & & & & \\
\hline 3. Income-to-needs ratio & $-.16^{*}$ & $-.17^{*}$ & - & & & \\
\hline $\begin{array}{l}\text { 4. Depressive symptoms } \\
\text { (CDI) }\end{array}$ & $.42^{* *}$ & .08 & -.09 & - & & \\
\hline $\begin{array}{l}\text { 5. Anhedonia symptoms } \\
\text { (MASQ-AD) }\end{array}$ & $.37^{* * *}$ & $.18^{\dagger}$ & -.08 & $.61^{* *}$ & - & \\
\hline 6. Anxiety symptoms (STAIC) & $.43^{* *}$ & .12 & -.10 & $.63^{* *}$ & $.47^{* *}$ & - \\
\hline \multicolumn{7}{|c|}{$\begin{array}{l}\text { QUIC Questionnaire of Unpredictability in Childhood, THQ Trauma History } \\
\text { Questionnaire, EPDS Edinburgh Postnatal Depression Scale, CTQ Childhood } \\
\text { Trauma Questionnaire, MASQ-AD Anhedonic Depression scale of the Mood } \\
\text { and Anxiety Symptom Questionnaire, CLES Coddington Life Events Scale, } \\
\text { CDI Children's Depression Inventory, STAIC State-Trait Anxiety Inventory for } \\
\text { Children } \\
{ }^{+} p<.10,{ }^{*} p<.05,{ }^{* *} p<.01\end{array}$} \\
\hline
\end{tabular}

needs ratio, exposure to stressful life events, parenting style, and household chaos.

This initial validation study is not without its limitations. First, our samples were predominantly Latino and non-Hispanic white and therefore it is critical that the QUIC's psychometric properties be examined in other populations including greater numbers of African American and Asian participants. Similarly, two of the three cohorts comprised only one gender and so further examination of potential gender differences also is warranted. In addition, both indicators of early-life adversity (e.g., traumatic life events) and mental health outcomes (e.g., depression) were assessed with different instruments and this limited our ability to compare the relative predictive validity across the three independent cohorts. However, this limitation is balanced by the fact that the findings were similar regardless of the assessment tools employed, increasing confidence that our findings are not specific to any particular instrument.

Increasing evidence supports the role of predictability of signals in early life as a potent contributor to lifespan health and development. Although self-report measures of specific aspects of unpredictability do exist (e.g., household chaos and family routines), until now, no comprehensive measure of unpredictability in childhood has been available. The QUIC's generalizability to populations other than those studied here remains to be established, but this new instrument's performance in the current study supports its promise for future use in both research and clinical contexts and for identification of new avenues for intervention.

\section{FUNDING AND DISCLOSURE}

This research was supported by grants from the National Institutes of Health (NS41298, HD51852, and MH96889), Veterans Affairs Center of Excellence for Stress and Mental Health, and Department of Defense CDMRP award MR141217.

\section{ACKNOWLEDGEMENTS}

We gratefully acknowledge the participants of these projects. 
Measuring novel antecedents of mental illness: the Questionnaire of... LM Glynn et al.

\begin{tabular}{|c|c|c|c|c|c|c|}
\hline Predictors & \multicolumn{2}{|c|}{$\begin{array}{l}\text { DV: adolescent depressive } \\
\text { symptoms }\end{array}$} & \multicolumn{2}{|c|}{$\begin{array}{l}\text { DV: adolescent anxiety } \\
\text { symptoms }\end{array}$} & \multicolumn{2}{|c|}{$\begin{array}{l}\text { DV: adolescent anhedonia } \\
\text { symptoms }\end{array}$} \\
\hline Age & $0.29^{\dagger}(0.17)$ & 0.13 & $0.66^{\dagger}(0.34)$ & 0.16 & $0.01(1.02)$ & 0.00 \\
\hline Sex & $1.28 *(0.58)$ & 0.16 & $3.68^{* *}(1.15)$ & 0.25 & $1.51(2.71)$ & 0.05 \\
\hline APQ Poor Monitoring/Supervision & $0.12(0.14)$ & 0.07 & $0.37(0.28)$ & 0.12 & $0.15(0.61)$ & 0.02 \\
\hline APQ Parental Involvement & $-0.37^{* *}(0.14)$ & -0.26 & $-0.19(0.27)$ & -0.07 & $-1.16^{\dagger}(0.64)$ & -0.23 \\
\hline APQ Corporal Punishment & $0.05(0.23)$ & 0.02 & $0.31(0.43)$ & 0.06 & $0.37(1.09)$ & 0.03 \\
\hline Income-to-needs ratio & $-0.00(0.00)$ & -0.09 & $0.00(0.00)$ & -0.01 & $0.00(0.01)$ & 0.00 \\
\hline Child Life Events (CLES) & $-0.08(0.08)$ & -0.08 & $-0.04(0.16)$ & -0.02 & $0.12(0.38)$ & 0.03 \\
\hline
\end{tabular}

APQ Alabama Parenting Questionnaire, CLES Childhood Life Events Scale, CHAOS Chaos, Hubbub, and Order Scale

${ }^{\dagger} p<.10,{ }^{*} p<.05,{ }^{* *} p<.01,{ }^{* * *} p<.001$

${ }^{\mathrm{a}}$ Coefficients are pooled estimates from the 10 imputed datasets

${ }^{\mathrm{b}}$ Coefficients were derived by averaging across the 10 imputed datasets. Complete case analyses were also performed and did not differ from results of analyses with imputed data reported here

\section{ADDITIONAL INFORMATION}

Supplementary Information accompanies this paper at (https://doi.org/10.1038/ s41386-018-0280-9).

Competing interests: The authors declare no competing interests.

Publisher's note: Springer Nature remains neutral with regard to jurisdictional claims in published maps and institutional affiliations.

\section{REFERENCES}

1. Repetti RL, Taylor SE, Seeman TE. Risky families: family social environments and the mental and physical health of offspring. Psychol Bull. 2002;128:330-66.

2. Belsky J. Early human experience: a family perspective. Dev Psychol. 1981;17:3-23.

3. Reiss F. Socioeconomic inequalities and mental health problems in children and adolescents: a systematic review. Soc Sci Med. 2013:90:24-31.

4. Heim C, Nemeroff CB. The role of childhood trauma in the neurobiology of mood and anxiety disorders: preclinical and clinical studies. Biol Psychiatry. 2001;49:1023-39.

5. Casey BJ, Ruberry EJ, Libby V, Glatt CE, Hare T, Soliman F, et al. Transitional and translational studies of risk for anxiety. Depress Anxiety. 2011;28:18-28.

6. Baram TZ, Davis EP, Obenaus A, Sandman CA, Small SL, Solodkin A, et al. Fragmentation and unpredictability of early-life experience in mental disorders. Am J Psychiatry. 2012;169:907-15.

7. Walker CD, Bath KG, Joels M, Korosi A, Larauche M, Lucassen PJ, et al. Chronic early life stress induced by limited bedding and nesting (LBN) material in rodents: critical considerations of methodology, outcomes and translational potential. Stress. 2017;20:421-48.

8. Molet J, Maras PM, Kinney-Lang E, Harris NG, Rashid F, Ivy AS, et al. MRI uncovers disrupted hippocampal microstructure that underlies memory impairments after early-life adversity. Hippocampus. 2016;26:1618-32.

9. Ivy AS, Rex CS, Chen Y, Dube C, Maras PM, Grigoriadis DE, et al. Hippocampal dysfunction and cognitive impairments provoked by chronic early-life stress involve excessive activation of CRH receptors. J Neurosci. 2010;30:13005-15.

10. Molet J, Heins K, Zhuo X, Mei YT, Regev L, Baram TZ, et al. Fragmentation and high entropy of neonatal experience predict adolescent emotional outcome. Transl Psychiatry. 2016;6:e702.

11. Bolton JL, Molet J, Regev L, Chen Y, Rismanchi N, Haddad E, et al. Anhedonia following early-life adversity involves aberrant interaction of reward and anxiety circuits and is reversed by partial silencing of amygdala corticotropin-releasing hormone gene. Biol Psychiatry. 2018;83:137-47.

12. Bolton JL, Ruiz CM, Rismanchi N, Sanchez GA, Castillo E, Huang J, et al. Early-life adversity faciliates acquisition of cocaine self-administration and induces persistent anhedonia. Neurobiol Stress. 2018;8:57-67.
13. Brunson KL, Kramar E, Lin B, Chen Y, Colgin LL, Yanagihara TK, et al. Mechanisms of late-onset cognitive decline after early-life stress. J Neurosci. 2005;25:9328-38.

14. Avishai-Eliner S, Eghbal-Ahmadi M, Tabachnik E, Brunson KL, Baram TZ. Downregulation of hypothalamic corticotropin-releasing hormone messenger ribonucleic acid (mRNA) precedes early-life experience-induced changes in hippocampal glucocorticoid receptor mRNA. Endocrinology. 2001;142:89-97.

15. Davis EP, Stout SA, Molet J, Vegetabile B, Glynn LM, Sandman CA, et al. Exposure to unpredictable maternal sensory signals influences cognitive development across species. Proc Natl Acad Sci USA. 2017;114:10390-5.

16. Belsky J, Schlomer GL, Ellis BJ. Beyond cumulative risk: distinguishing harshness and unpredictability as determinants of parenting and early life history strategy. Dev Psychol. 2012;48:662-73.

17. Ellis BJ, Figueredo AJ, Brumbach BH, Schlomer GL. Fundamental dimensions of environmental risk: the impact of harsh versus unpredictable environments on the evolution and development of life history strategies. Hum Nat. 2009;20:204-68.

18. Priel A, Djalovski A, Zagoory-Sharon O, Feldman R. Maternal depression impacts child psychopathology across the first decade of life: oxytocin and synchrony as markers of resilience. J Child Psychol Psychiatry. 2018. https://doi.org/10.1111/ jcpp. 12880.

19. Bernard K, Meade EB, Dozier M. Parental synchrony and nurturance as targets in an attachment based intervention: building upon Mary Ainsworth's insights about mother-infant interaction. Attach Hum Dev. 2013;15:507-23.

20. Ainsworth MDS, Bowlby J. An ethological approach to personality development. Am Psychol. 1991;46:333-41.

21. Evans GW, Gonnella C, Marcynyszyn LA, Gentile L, Salpekar N. The role of chaos in poverty and children's socioemotional adjustment. Psychol Sci. 2005;16:560-5.

22. Manczak EM, Williams D, Chen E. The role of family routines in the intergenerational transmission of depressive symptoms between parents and their adolescent children. J Abnorm Child Psychol. 2017;45:643-56.

23. Doom JR, Vanzomeren-Dohm AA, Simpson JA. Early unpredictability predicts increased adolescent externalizing behaviors and substance use: a life history perspective. Dev Psychopathol. 2016;28:1505-16.

24. Simpson JA, Griskevicius V, Kuo SI, Sung S, Collins WA. Evolution, stress, and sensitive periods: the influence of unpredictability in early versus late childhood on sex and risky behavior. Dev Psychol. 2012;48:674-86.

25. Glynn LM, Howland MA, Sandman CA, Davis EP, Phelan M, Baram TZ, et al. Prenatal maternal mood patterns predict child temperament and adolescent mental health. J Affect Disord. 2018;228:83-90.

26. Hooper LM, Stockton P, Krupnick JL, Green B. Development, use, and psychometric properties of the Trauma History Questionnaire. J Loss Trauma. 2011;16:258-83.

27. Cox JL, Holden JM, Sagovsky R. Detection of postnatal depression. Development of the 10-item Edinburgh Postnatal Depression Scale. $\mathrm{Br} J$ Psychiatry. 1987;150:782-6. 
Measuring novel antecedents of mental illness: the Questionnaire of...

LM Glynn et al.

882

28. Baker DG, Nash WP, Litz BT, Geyer MA, Risbrough VB, Nievergelt CM, et al. Predictors of risk and resilience for posttraumatic stress disorder among ground combat Marines: methods of the Marine Resiliency Study. Prev Chronic Dis. 2012;9:E97.

29. Bernstein DP, Fink L, Handelsman L, Foote J, Lovejoy M, Wenzel K, et al. Initial reliability and validity of a new retrospective measure of child abuse and neglect. Am J Psychiatry. 1994;151:1132-6.

30. Watson D, Clark LA. The Mood and Anxiety Symptom Questionnaire. lowa City, IA: Department of Psychology, University of lowa; 1991.

31. Kendall AD, Zinbarg RE, Bobova L, Mineka S, Revelle W, Prenoveau JM, et al. Measuring positive emotion with the Mood and Anxiety Symptom Questionnaire: psychometric properties of the Anhedonic Depression Scale. Assessment . 2016;23:86-95.

32. Spielberger CD, Edwards CD, Montouri J, Lushene R. The State-Trait Anxiety Inventory for Children. Palo Alto, CA: Consulting Psychologist Press; 1973.
33. Kovacs M. Children's Depression Inventory 2 (CDI 2). 2nd ed. North Tonawanda, NY: Multi-Health Systems Inc; 2011.

34. Shelton KK, Frick PJ, Wootton J. Assessment of parenting practices in families of elementary school-age children. J Clin Child Psychol. 1996;25:317-29.

35. Coddington RD. The significance of life events as etiologic factors in the diseases of children. A survey of professional workers. J Psychosom Res. 1972;16:7-18.

36. Matheny AP, Wachs TD, Ludwig JL, Phillips K. Bringing order out of chaos: psychometric characteristics of the Confusion, Hubbub, and Order Scale. J Appl Dev Psychol. 1995;16:429-44.

37. Rubin DB. Multiple imputation for nonresponse in surveys. New York, NY: John Wiley \& Sons; 1987.

38. Azur MZ, Stuart EA, Frangakis C, Leaf PJ. Multiple imputation by chained equations: what is it and how does it work? Int J Methods Psychiatr Res. 2011;20:40-9.

39. Streiner DL. Starting at the beginning: an introduction to coefficient alpha and internal consistency. J Pers Assess. 2003;80:99-103. 